\title{
Sintomas de internalização e externalização em crianças e adolescentes com excesso de peso
}

\author{
Internalizing and externalizing symptoms in children \\ and adolescents with overweight \\ Marcos Rogério Capello Sousa', César de Moraes
}

\section{RESUMO}

Objetivo: Comparar sintomas de internalização e externalização em crianças e adolescentes com e sem excesso de peso. Método: 88 indivíduos (53 com excesso de peso e 35 eutróficos), entre 6 e 18 anos, foram avaliados por meio do Inventário de Comportamentos da Infância e Adolescência (CBCL-6/18) e de medidas antropométricas. Foram considerados com excesso de peso os indivíduos com índice de massa corporal (IMC) > 1 desvio-padrão. Os indivíduos com T-score > 70 no CBCL-6/18 foram considerados como tendo sintomas emocionais. O teste de Mann-Whitney foi utilizado para verificar se houve diferença entre as médias do IMC, as médias de idade e os T-scores médios obtidos nas subescalas de internalização e externalização, de acordo com os grupos pesquisados. Foi utilizado o índice de correlação de Spearman para verificar correlação entre o IMC e os T-scores médios obtidos nas subescalas de internalização e externalização. Resultados: Sintomas de internalização ocorreram em 14 indivíduos com excesso de peso e em 4 eutróficos (médias de T-score: 59,09 e 49,40, respectivamente, $p$-valor $=0,003$ ). Sintomas de externalização ocorreram em 9 indivíduos com excesso de peso e em 2 eutróficos (médias de T-score: 53,71 e 47,91, respectivamente, p-valor =0,019). Foi encontrada uma correlação positiva entre o IMC dos indivíduos pesquisados e a presença de sintomas de internalização e externalização. O mesmo ocorreu, quando se correlacionou os T-scores médios dos sintomas de internalização e externalização. Conclusão: Sintomas psiquiátricos prevaleceram nos indivíduos com excesso de peso. Quanto maior é o IMC, maior o risco do desenvolvimento de sintomas emocionais.

\begin{abstract}
Objective: The aim of this study was to compare the internalizing and externalizing symptoms in children and adolescents with and without weight excess. Method: Child Behavior Checklist (CBCL-6/18) and Body Mass Index (BMI) have been used to assess 88 individuals from 6 to 18 years of age. The individuals with BMI > 1 sd were considered overweight (53 overweight and 35 eutrophic) and those individuals with CBCL-6/18 T-score > 70 were considered having emotional symptoms. The Mann-Whitney test was used to measure any possible difference in the average BMI, between age averages and mean t-scores obtained in internalizing and externalizing subscales according to the researched groups. Spearman Correlation Index was used to verify the correlation between BMI and T-score averages. Results: Internalizing symptoms have been observed in 14 overweight
\end{abstract}

Recebido em
$8 / 11 / 2009$
Aprovado em
24/1/2011

1 Universidade Estadual de Campinas (Unicamp). Trabalho desenvolvido nas UBS do Jd. Florence e Jd. Rossin, Campinas, SP, por meio de parceria entre a Pontifícia Universidade Católica de Campinas e a prefeitura municipal de Campinas.

Endereço para correspondência: César de Moraes Rua Alvaro Bosco, 157, ap. 31-B 3087-723 - Campinas, SP Telefone: (19) 9219-8987 - Fax: (19) 3236-2487

E-mail: cemoraes@uol.com.br 


\section{Keywords}

Obesity, childhood, adolescence, behavior disorder. individuals and in 4 eutrophic individuals. (T-score average: 59.09 and 49.40, respectively, $p$ value $=0.003$ ). Externalizing symptoms have been found in 9 overweight individuals and in 2 eutrophic individuals. (T-score average: 53.71 and 47.91, respectively, $p$-value $=0.019$ ). There was a positive correlation between BMl and internalizing and externalizing symptoms. Conclusion: Psychiatric symptoms have been observed frequently in overweight individuals and the higher is the BMI, the higher is the risk for developing emotional symptoms.

\section{INTRODUÇÃO}

O excesso de peso na infância e adolescência é um problema mundial, acometendo países desenvolvidos e em desenvolvimento 1,2. A Organização Mundial de Saúde (OMS) estima que 10\% dos indivíduos entre 5-17 anos estão com sobrepeso e 2\%-3\% estão com obesidade ${ }^{3}$. Até 2005 havia 20 milhões de crianças menores de 5 anos com excesso de peso $0^{4}$. Monteiro et al. ${ }^{5}$ encontraram prevalência de $20,5 \%$ para sobrepeso e 7,7\% para obesidade entre adolescentes (14-16 anos). Seu surgimento precoce aumenta a possibilidade da obesidade no adulto (fenômeno da trilha ou tracking)* ${ }^{*}$, antecipando doenças cujas taxas de morbimortalidade já contabilizam 59\% das 57 milhões de mortes anuais e $46 \%$ de todas as doenças. O Ministério da Saúde ${ }^{7}$ registrou 43,4\% de adultos com excesso de peso e $27 \%$ de mortes por doença cardiovascular 8, , independentemente da idade. Condições patológicas frequentes ligadas à obesidade como diabetes tipo II, transtornos emocionais - bullying, ansiedade, depressão ${ }^{10}$ - e osteoartropatias ${ }^{11}$ podem surgir subitamente com o agravamento de doença sem controle ou sem diagnóstico, suscitando tratamento emergencial especializado e, posteriormente, reabilitação para reintegração social. Esse cenário de intervenções curativas e de reabilitação com reintegração social põe em risco os sistemas de saúde e previdenciários de países desenvolvidos, mas principalmente aqueles de países em desenvolvimento ${ }^{12}$.

A gênese da obesidade infanto-juvenil parece mais ligada às mudanças ambientais relacionadas à organização da economia capitalista mundial nos últimos 40 anos $^{13-15}$. Estas têm impacto nas mudanças de comportamento e na cultura das comunidades, escolas, lares e locais de trabalho, mas ainda há pouca investigação ${ }^{16}$ de mediações como aquela que se dá entre a realidade e a subjetividade dos indivíduos, principalmente as crianças, e que é feita pela mídia por meio de propagandas abusivas incentivando o consumo e o lazer sedentário ${ }^{17}$. Nessa perspectiva, para dimensionar o impacto da obesidade na saúde coletiva e propor intervenções, a avaliação do comportamento infanto-juvenil torna-se imperativa ao lado das avaliações dietéticas e da prática de exercício físico. Para isso, são necessários instrumentos validados que identifiquem se os distúrbios psíquicos comportam-se

\footnotetext{
* Condições de risco associadas à obesidade como hipertensão arterial, dislipidemia e resistência insulínica, que, iniciadas durante a infância-adolescência, tendem a se expressar na vida adulta.
}

como causa, consequência ou um fator associado à obesidade. Sabe-se que indivíduos com depressão frequentemente apresentam estilo de vida precário por causa consumo excessivo de fumo, álcool, dietas não saudáveis e sedentarismo e apresentam baixa adesão aos tratamentos médicos. Mas, mesmo na ausência desses fatores, existem alterações fisiológicas nos quadros depressivos que poderiam responder pelas elevadas taxas de doenças cardiovasculares nesses indivíduos como os mecanismos autonômicos de regulação da frequência cardíaca, a desregulação do eixo hipotálamo-pituitária-adrenal e a perda da modulação dos processos inflamatórios e hemostáticos ${ }^{18}$.

Estudos populacionais correlacionando disfunções psíquicas e obesidade em crianças e adolescentes ainda são escassos, mas, mesmo não permitindo generalizações nem explicando mecanismos mediadores entre obesogênese e psicopatias na população infanto-juvenil, eles têm demonstrado aumento na prevalência de distúrbios psíquicos nessas faixas etárias. Isso, por si só, evidencia a importância da inclusão de avaliações psiquiátricas validadas em atendimentos especializados ${ }^{19}$. Qualquer que seja a relação entre obesidade e transtorno mental, o objetivo de verificar problemas emocionais em crianças e adolescentes obesos por meio desses inquéritos é, além de construir conhecimento científico que subsidie as ações assistenciais, identificar precocemente os indivíduos cujos comportamentos de risco interfiram nas intervenções e prevenção do fenômeno da trilha (tracking) ${ }^{6}$.

Segundo Achenbach ${ }^{20}$, manifestações emocionais em crianças e adolescentes podem ser divididas em dois grandes grupos: os sintomas de internalização e os sintomas de externalização.

Sintomas de internalização são aqueles percebidos pela criança e adolescente de forma subjetiva ou física, sem uma manifestação comportamental, necessariamente, associada a ele. Sintomas de ansiedade, depressão, retraimento, além das manifestações somáticas, são exemplos de sintomas desse grupo.

Por outro lado, os sintomas de externalização são aqueles manifestados de forma claramente comportamental por meio de atos motores. A agressividade e o comportamento delinquente são exemplos de sintomas desse grupo.

De forma geral, sintomas de externalização geram maior impacto negativo sobre o ambiente. Já os sintomas de in- 
ternalização geram maior sofrimento emocional e subjetivo para a própria pessoa.

O objetivo deste estudo é comparar a frequência de sintomas psiquiátricos de internalização e externalização em indivíduos com e sem excesso de peso, numa população infanto-juvenil usuária de unidade básica de saúde.

\section{MÉTODOS}

Foi utilizado um método quantitativo, transversal, com grupo controle.

\section{Sujeitos}

Foram avaliados 88 crianças e adolescentes, com idade entre 6 e 17 anos, divididas em dois grupos: 53 indivíduos com excesso de peso e 35 indivíduos eutróficos.

Os indivíduos foram selecionados nas Unidades Básicas de Saúde (UBS), conveniadas com a PUC-Campinas, ou no ambulatório de pediatria geral do Hospital Universitário da referida universidade. Esses serviços atendem a uma região de periferia da cidade de Campinas, SP.

Baseados nos critérios da OMS $21-23$, foram incluídos no grupo de excesso de peso os indivíduos com índice de massa corporal (IMC) maior que 1 e 2 desvios-padrão (dp). Foram incluídos no grupo de eutróficos indivíduos com IMC menor que 1 dp e maior que -2 dp.

Foram excluídos crianças e adolescentes com obesidade de causa endógena, em uso de medicações que promovem ganho de peso, gestantes ou qualquer outra situação clínica pediátrica que interferisse na aferição precisa do peso e estatura para cálculo do IMC. Foram realizadas avaliações pediátricas para descartar outras patologias que favoreçam o ganho de peso.

\section{Instrumentos utilizados}

A aferição dos dados antropométricos foi realizada com roupas leves; a estatura foi medida em estadiômetro de parede (Sanny) e o peso, em balança digital (Filizola).

A avaliação dos sintomas comportamentais foi realizada por meio do Childhood Behavior Checklist 6-18 (CBCL-6/18). O instrumento é um questionário que avalia áreas de competência e sintomas de internalização e externalização em crianças e adolescentes de 6 a 18 anos, a partir de informações fornecidas pelos pais.

Desenvolvido por Achenbach ${ }^{20}$, destaca-se dentre os questionários de comportamentos mais citados na literatura americana, não somente pelo rigor metodológico com que foi elaborado, como também por sua utilidade na prática clínica e aplicação em trabalhos científicos. Pode ser utilizado em escolas e em serviços de saúde mental, por seu poder de discriminar indivíduos com psicopatologia de indivíduos normais.
Traduzido em mais de 30 idiomas, tem sido empregado internacionalmente em pesquisas envolvendo epidemiologia, diagnóstico, taxonomia, etiologia, tratamento e comparações entre culturas. Foi validado para a realidade brasileira por Bordin et al., em 199524.

Para efeito de análise, foram utilizadas as subescalas de Internalização e Externalização do CBCL-6/18, sendo considerados indivíduos sintomáticos aqueles que obtiveram um resultado de T-score maior ou igual a 70 pontos.

Foi aplicada uma anamnese pediátrica estruturada para coleta de antecedentes, problemas clínicos associados e outros critérios de exclusão.

\section{Procedimentos experimentais gerais}

Após assinatura do Termo de Consentimento Livre e Esclarecido (aprovado pelo Comitê de Ética em Pesquisa da PUC-Campinas) pelos pais ou responsáveis dos indivíduos pesquisados (e também por aqueles adolescentes que tinham clara compreensão dos propósitos e encaminhamentos da pesquisa), foram selecionados os sujeitos, segundo os critérios de inclusão e exclusão, e divididos os grupos, de acordo com os resultados dos dados antropométricos. Depois da seleção dos sujeitos, os seus pais foram convidados para uma entrevista. Nesse momento, foram respondidos os instrumentos de avaliação (CBCL-6/18 e anamnese estruturada).

\section{Análise estatística}

Além da estatística descritiva, foi realizado o teste exato de Fischer para avaliar as variáveis categoriais, como a proporção de homens e mulheres e a presença de sintomas de internalização e externalização entre os indivíduos dos diferentes grupos pesquisados.

O teste de Mann-Whitney foi utilizado para verificar se houve diferença entre as médias do índice de massa corporal (IMC), as médias de idade e os T-scores médios obtidos nas subescalas de internalização e externalização, de acordo com os grupos pesquisados.

Foi utilizado o índice de correlação de Spearman para verificar se houve correlação entre o IMC e os T-scores médios obtidos nas subescalas de internalização e externalização.

Para todos os testes utilizados, foi considerado um nível de significância com p-valor $<0.05$.

\section{RESULTADOS}

Entre os 53 indivíduos do grupo de obeso/sobrepeso, havia 31 do sexo masculino e 22 do sexo feminino, com idade média de 10,02 \pm 2,53 anos e IMC médio de 25,65 \pm 5,46. Indivíduos controles eutróficos foram 35, sendo 14 do sexo masculino e 21 do sexo feminino, com idade média de $11,03 \pm 3,04$ anos e IMC médio de 17,37 $\pm 2,31$. Não houve diferença estatisticamente significativa em relação às 
médias de idade e a proporção de homens e mulheres nos grupos pesquisados.

Do total dos indivíduos estudados, 18 (20,6\%) apresentaram sintomas de internalização e 11 (12,64\%), de externalização. O T-score médio para sintomas de internalização foram significativamente maiores entre os indivíduos obesos/ sobrepeso do que entre os indivíduos eutróficos. O mesmo ocorreu com o T-score médio para sintomas de externalização (Tabela 1).

Tabela 1. Descrição das variáveis, de acordo com grupo pesquisado

\begin{tabular}{llll}
\hline & Eutrófico & Obeso/sobrepeso & p-valor \\
\hline $\mathrm{N}$ & 35 & 53 & \\
Idade & $11,03 \pm 3,04$ & $10,02 \pm 2,53$ & 0,119 \\
Sexo & & & 0,069 \\
$\quad$ Masculino & 14 & 31 & \\
$\quad$ Feminino & 21 & 22 & $\mathbf{0 , 0 0 0}$ \\
IMC & $17,37 \pm 2,31$ & $25,65 \pm 5,46$ & 0,003 \\
$\begin{array}{l}\text { Sintomas de internalização } \\
\text { (T-score) }\end{array}$ & $49,40 \pm 14,20$ & $59,09 \pm 12,61$ & \\
$\begin{array}{l}\text { Sintomas de externalização } \\
\text { (T-sccore) }\end{array}$ & $47,91 \pm 11,08$ & $53,71 \pm 11,67$ & 0,019 \\
\hline Os dados são apresentados comomédia \pm desvio-padrão (SD) exceto onde especificado. & \\
\hline
\end{tabular}

Os dados são apresentados como média \pm desvio-padrão (SD), exceto onde especificado.

Quando se considera um T-score médio > 70 para descrever indivíduos sintomáticos, nota-se que, dentre indivíduos com excesso de peso, 17 (32,07\%) apresentaram sintomas psiquiátricos de internalização e/ou externalização. O mesmo ocorreu em 5 indivíduos (14,7\%) do grupo dos eutróficos ( $p$-valor $=0,49)$. Dentre os indivíduos com sintomas de internalização, 14 (77,7\%) estavam acima do peso e apenas $4(24,3 \%)$ eram eutróficos ( $p$-valor $=0,73)$. Dentre os indivíduos com sintomas de externalização, $9(81,8 \%)$ apresentavam excesso de peso e $2(18,2 \%)$ estavam eutróficos ( $p$-valor $=0,106)$.

Foi utilizada uma análise de correlação para avaliar a relação entre a gravidade do excesso de peso e os sintomas de internalização e externalização. O IMC se correlacionou positivamente com os sintomas de internalização $(r=0,374$, $p<0,001)$ e de externalização $(r=0,217 ; p=0,042)$. Além disso, os sintomas de internalização também se correlacionaram positivamente com os sintomas de externalização $(r=0,767, p<0,001)$.

\section{DISCUSSÃO}

O estudo identificou, por meio do $\mathrm{CBCL}$, maior frequência de sintomas de internalização e externalização em indivíduos com excesso de peso.
Há vários estudos na literatura utilizando o CBCL para verificar o comportamento de crianças e adolescentes tanto em grupos com doenças definidas quanto em grupos com perfil de risco para surgimento de doenças psíquicas.

Vila et al. ${ }^{19}$ utilizaram o CBCL como um de seus instrumentos de pesquisa para verificação de sintomas emocionais em crianças e adolescentes franceses obesos comparados com um grupo de diabéticos. Os autores encontraram taxas de sintomas de internalização e externalização bem maiores que as encontradas em nosso estudo: $43 \%$ dos indivíduos obesos estudados apresentaram sintomas de internalização e 25\%, de externalização. Neste trabalho, 26\% dos indivíduos obesos apresentaram sintomas de internalização e 17\% apresentaram sintomas de externalização. Ou seja, as taxas de sintomas de internalização e externalização foram duas (internalização) a três vezes (externalização) maiores no grupo com excesso de peso se comparado ao grupo de indivíduos eutróficos.

Outros estudos utilizaram instrumentos diagnósticos, em vez de instrumentos de verificação de sintomas (como CBCL), para a detecção de transtornos psiquiátricos em indivíduos obesos. Estudando indivíduos canadenses com 15 anos ou mais, Mather et al. ${ }^{25}$ relataram que a obesidade se relaciona positivamente com uma série de patologias psiquiátricas, a saber, depressão, mania, ataques de pânico, fobia social e ideação suicida. Alguns estudos têm verificado a presença de patologias psiquiátricas em indivíduos obesos adultos ${ }^{26}$.

Petry et al. ${ }^{27}$ encontraram taxas maiores de transtornos de ansiedade e abuso de substâncias psicoativas entre indivíduos obesos, mas não encontraram taxas maiores de depressão. Apesar de a maioria desses estudos ter sido realizada com adultos, eles apresentaram resultados similares aos nossos, isto é, demonstraram que os transtornos depressivos e de ansiedade são mais prevalentes na população de indivíduos obesos. Em nosso estudo, as altas taxas de sintomas de internalização observadas por meio do $C B C L$ se referem fundamentalmente a sintomas de ansiedade, depressão e retraimento social.

Por outro lado, apesar de não haver relatos da presença de sintomas de externalização nos estudos revisados (comportamento agressivo e comportamento delinquente), eles aparecem, em nosso estudo, em indivíduos que também apresentavam sintomas de internalização. Em sua grande parte, essa associação de sintomas ocorreu em meninas, apesar de os sintomas de externalização serem quase duas vezes mais frequentes em meninos do que em meninas ${ }^{28}$. Esse fato pode indicar maior gravidade dos transtornos emocionais e comportamentais em meninas. Os sintomas de externalização e internalização puros ocorreram apenas entre os meninos.

Percebeu-se uma clara correlação entre a presença de sintomas de depressão, de ansiedade e de comportamentos disruptivos com um maior IMC dos indivíduos pesquisados. 
Isso demonstra que a presença desses sintomas pode ser um fator de risco para a presença de obesidade, por cursar com aumento de apetite, redução da atividade física e maior ingesta de alimentos calóricos (como chocolates e doces). Por outro lado, uma autoimagem negativa (e, consequentemente, uma baixa autoestima), decorrente da presença da obesidade, pode gerar sintomas emocionais como dificuldades de relacionamento social, maior isolamento, alterações de conduta e comportamento opositor.

Além disso, o estudo demonstra que a presença de um grupo de sintomas pesquisados (internalização ou externalização) favorece a presença do outro grupo de sintomas pesquisados. Por exemplo, o indivíduo obeso que apresentar um grupo de sintomas depressivos e ansiosos (sintomas de internalização) tem uma chance aumentada de apresentar também sintomas disruptivos (sintomas de externalização: furtar, mentir, agredir). $\mathrm{O}$ estudo de Bruffaerts et al. ${ }^{26}$, com a população geral de seis países europeus, reforça esse fato. Eles encontraram que os indivíduos obesos costumam apresentar mais de uma patologia psiquiátrica e que a depressão é a principal delas.

Uma das principais limitações dessa pesquisa foi a utilização de um instrumento pautado na resposta dos pais, sem que as crianças e adolescentes tivessem passado por uma avaliação posterior para confirmação dos sintomas e pesquisa de possíveis diagnósticos. Segundo Bordin e Paula ${ }^{29}$, as taxas de problemas de saúde mental entre crianças e adolescentes brasileiros, quando são considerados os pais como únicos informantes, são de 13,5\% a 35,2\%, enquanto nos estudos que utilizam os adolescentes como únicos informantes essas taxas diminuem para $12,6 \%$ a $13,1 \%$. Novos estudos que utilizem métodos de seguimento ao longo do desenvolvimento infanto-juvenil, e não apenas estudos transversais, e que pesquisem os sintomas emocionais e comportamentais de acordo com a gravidade da obesidade são necessários.

\section{CONCLUSÕES}

Sintomas psiquiátricos de internalização e externalização são mais comuns entre crianças e adolescentes com excesso de peso do que entre indivíduos eutróficos. A proposta de utilizar o CBCL para triagem de crianças e adolescentes com excesso de peso e sintomas psíquicos torna-se relevante, pois se trata de um instrumento validado e que pode facilitar o trabalho do médico pediatra ao abordar a criança e o adolescente com excesso de peso e propor um programa de reeducação alimentar e de atividade física, tendo em conta que transtornos do comportamento podem comprometer intervenções preventivas e curativas.

Os resultados obtidos a partir do trabalho integrado de duas áreas médicas - pediatria e psiquiatria infantil - mostram a necessidade de readequarmos nossas abordagens diagnósticas para tentar interromper a gênese da obesidade na população infanto-juvenil.

\section{REFERÊNCIAS}

1. WHO: Regional Office for Europe. The challenge of obesity in the WHO European Region and the strategies for response. Disponivel em: <http://www.euro.who.int/pubrequest>. Acesso em: 17 dez 2007.

2. Silveira D, Taddei JA, Escrivão MA, Oliveira FL, Ancona-Lopez F. Risk factors for overweight among Brazilian adolescents of low-income families: a case-control study. Public Health Nutr. 2006;9(4):421-8.

3. WHO. Facts related chronic diseases. Disponivel em: http://www.who.int/dietphysicalactivity/publications/facts/chronic/en/. Acesso em: 9 jun 2004.

4. WHO. Obesity: preventing and managing the global epidemic. Report of a WHO Consultation on 0besity. Geneva; 1998. Disponível em: <www.who.int/childgrowth/>. Acesso em: 17 dez 2007.

5. Monteiro POA, Victora CG, Barros FC, Monteiro LMA. Birth size, early childhood growth, and adolescent obesity in a Brazilian birth cohort. Int I Obesity. 2003;27(10):1274-82.

6. Tan F, Okamoto M, Suyama A, Miyamoto T. Tracking of cardiovascular risk factors and cohort study on hyperlipidemia in rural schoolchildren in Japan. J Epidemiol. 2000;10(4):255-61.

7. Ministério da Saúde. Portal da Saúde. Mapa da Saúde. Disponível em: <http://www.saude.gov.br/>. Acesso em: 7 abr. 2008.

8. Ministério da Saúde. DATASUS. Informaçōes sobre mortalidade, informações demográficas. Disponível em: <http://tabnet.datasus.gov.br>. Acesso em: 10 abr. 2008.

9. Godoy MF, Lucena JM, Miquelin AR, Paiva FF, Oliveira DLQ, Augustin Jr JL. Mortalidade por doenças cardiovasculares e níveis socioeconômicos na população de São José do Rio Preto, Estado de São Paulo, Brasil. Arq Bras Cardiol. 2007;88(2):200-6.

10. Ross CE. Overweight and depression. J Health Soc Behav. 1994;35:63-79.

11. Fisberg M. Atualização em obesidade na infância e adolescência. São Paulo: Atheneu; 2005.

12. Kottke TE, Wu LA, Hoffman RS. Economic and psychological implications of the obesity epidemic. Mayo Clin Proc. 2003;78(1):92-4.

13. Bordo AA. Os eixos de desenvolvimento e a estruturação urbano-industrial do estado de São Paulo, Brasil. Scripta Nova. Revista Electrônica de Geografía y Ciencias Sociales. Barcelona. Universidade de Barcelona, agosto, 2005 vol IX, 194(79). Disponível em: <http:// www.ub.es/geocrit/sn-194-79.htm>. Acesso em: 12 set. 2006.

14. Anais XII Simpósio Brasileiro de Sensoriamento Remoto. Jakob AAE, Cunha JMP. Delimitação de áreas de segregação espacial da população na mancha urbana da região metropolitana de Campinas a partir de imagens de satélite e técnicas de estatística espacial. Disponível em: <http://www.ub.es/geocrit/sn-194-79.htm. Acesso em: 12 set. 2006.

15. Kumanyika S, Jeffery RW, Morabia A, Ritenbaugh C, Antipatis VJ; Public Health Approaches to the Prevention of Obesity (PHAPO). Working Group of the International Obesity Task Force (IOTF). Obesity prevention: the case for action. Int J Obesity. 2002;26(3):425-36.

16. Dressler WW, Santos JE. Social and cultural dimensions of hypertension in Brazil: review. Cad Saude Publica. 2000;16(2):303-15.

17. Miotto AC, Oliveira AF. A influência da mídia nos hábitos alimentares de crianças de baixa renda. Rev Paul Pediatr. 2006;24(2):115-20.

18. Kinder LS, Carnethon MR, Palaniappan LP, King AC, Fortmann SP. Depression and the metabolic syndrome in young adults: findings from the Third National Health and Nutrition Examination Survey. Psychosom Med. 2004;66:316-22.

19. Vila G, Zipper E, Dabbas M, Bertrand C, Robert JJ, Ricour C, et al. Mental disorders in obese children and adolescents. Psychosom Med. 2004;66:387-94.

20. Achenbach TA. Manual for the Child Behavior Checklist/4-18 and 1991 Profile. Burlington, VT: University of Vermont, Department of Psychiatry; 1991.

21. Onis M, Onyango AW, Borghi E, Siyam A, Nishida C, Siekmann J. Development of a WHO growth reference for school-aged children and adolescents. Bull World Health Organ. 2007;85(9):660-7. 
22. World Health Organization (WHO). WHO Child Growth Standards Training Course on Child Growth. Disponível em: <www.who.int/childgrowth/>. Acesso em: 18 set. 2007.

23. World Health Organization (WHO). Disponível em: <http://www.who.int/ageing/en/>. Acesso em: $1^{0}$ abr. 2008

24. Bordin IAS, Mari JJ, Caieiro MF. Validade da versão brasileira do "Child Behavior Checklist" (CBCL) (Inventário de Comportamentos da Infância e Adolescência): dados preliminares. Rev ABP-APAL. 1995;17(2):55-66.

25. Mather AA, Cox BJ, Enns MW, Sareen J. Associations of obesity with psychiatry disorder and suicidal behavior in a nationally representative sample. J Psychom Res. 2009;66:277-85.
26. Bruffaerts R, Demyttenaere K, Vilagut G, Martinez M, Bonnewyn A, De Graeff R, et al. The relation between body mass index, mental health, functional disability: a European population perspective. Can J Psychiatry. 2008;50(10):679-88.

27. Petry N, Barry D, Pietrzak RH, Wagner J. Overweight and obesity are associated with psychiatric disorders: results from the National Epidemiologic Survey on Alcohol and related conditions. Psychosom Med. 2008;70:288-97.

28. Nock MK, Kazdin AE, Hiripin E, Kessler, RC. National Comorbidity Survey Replication. Psychol Med. 2006;36 (5):699-710.

29. Bordin IAS, Paula CS. Estudos populacionais sobre saúde mental de crianças e adolescentes. In: Mello MF, Mello AAF, Kohn R, organizadores. Epidemiologia da Saúde Mental no Brasil. São Paulo: Artmed; 2007 